\title{
Impaired mucosal immunity in patients with SLE
}

A new study published this week in The Journal of Immunology identifies deficiencies in mucosal-associated invariant T (MAIT) cells from patients with systemic lupus erythematosus (SLE).

MAIT cells have an important function in mucosal immunity by protecting against mycobacterial and enterobacterial infections. In turn, microbial infections have been implicated as environmental factors that can influence autoimmune diseases in genetically predisposed individuals. "The role of MAIT cells remains enigmatic in autoimmune diseases," explains Yong-Wook Park from Chonnam National University, the corresponding author of the study.

To establish the clinical relevance of MAIT cells in rheumatic diseases, Park and colleagues isolated peripheral blood mononuclear cells from patients with SLE $(n=54)$, rheumatoid arthritis (RA, $n=66)$, ankylosing spondylitis $(n=21)$ or Behçet syndrome $(n=9)$, and healthy individuals $(n=136)$. Both the frequency and number of MAIT cells (defined as CD3 ${ }^{+}$TCR $\gamma \delta^{-}$ Va7. $\left.2^{+} \mathrm{CD} 161^{\text {high }}\right)$ were reduced in patients with SLE $(0.29 \%$ vs $2.13 \%, P<0.001 ; 0.9$ vs 16.2 cells/ $\mu \mathrm{l}, P<0.001)$ or RA $(0.73 \%$ vs $2.13 \%, P<0.01 ; 3.1$ vs 16.2 cells per $\mu \mathrm{l}$, $P<0.05)$ when compared with healthy controls. No differences were observed in patients with ankylosing spondylitis or Behçet syndrome.

Importantly, this decrease in circulating MAIT cells correlated with SLE and RA disease activity according to SLE disease activity index score or 28-joint disease activity score, respectively. MAIT cells isolated from patients with SLE (but not cells isolated from patients with RA) showed decreased activation as measured by the frequency of IFN- $\gamma$-positive cells $(32.1 \%$ vs $53.0 \%, P<0.005)$ upon in vitro stimulation when compared with MAIT cells from healthy individuals, a defect associated with reduced nuclear translocation of nuclear factor of activated T cells, cytoplasmic 2
(NFAT1) and increased expression of the coinhibitory molecule programmed cell death protein 1 (PD-1).

\section{4 ...decrease in circulating MAIT cells correlated with SLE and RA disease activity... 77}

"Our study was the first to demonstrate that MAIT cells are numerically and functionally deficient in SLE, and that MAIT cell deficiency reflects disease activity," adds Park, whereas in patients with RA, "numerical deficiency but preserved cytokine production in MAIT cells ... may be due to migration of MAIT cells into inflamed joints".

João H. Duarte

Original article Cho, Y.-N. et al. Mucosal-associated invariant T cell deficiency in systemic lupus erythematosus. J. Immunol. doi:10.4049/jimmunol.1302701 